\title{
Antistreptolysin O Positivity in Chronic Tonsillitis: Rare or Common?
}

\author{
${ }^{1}$ UP Santosh, ${ }^{2} \mathrm{~KB}$ Prashanth, ${ }^{3}$ Nitha Mary George
}

\section{ABSTRACT}

Objective: To determine the frequency of antistreptolysin $\mathrm{O}$ (ASLO) positivity in cases of proven chronic tonsillitis.

Materials and methods: The present study was conducted in a tertiary care center from January to October 2013. A total of 150 patients who presented with features suggestive of chronic tonsillitis and willing for surgery were included in the study. On admission, following a detailed history taking and clinical examination, preoperative investigations along with ASLO titers were done. Following medical and anesthetic fitness, the patients underwent tonsillectomy with or without adenoidectomy and the tonsillar specimens were sent for histopathological examination.

Results: A total of five out of the 150 patients showed ASLO titer positivity (>200 IU/ml) and all were histopathologically proven cases of chronic tonsillitis.

Conclusion: Early detection and treatment of Streptococcal tonsillar involvement could be a preventive measure against the future predilection of other predisposed organs to more severe and debilitating streptococcal infections.

Keywords: Chronic tonsillitis, Rheumatic factor, Antistreptolysin $\mathrm{O}$ antibodies, Rheumatic fever, Streptococcal infection, Tonsillectomy.

How to cite this article: Santosh UP, Prashanth KB, George NM. Antistreptolysin O Positivity in Chronic Tonsillitis: Rare or Common? Int J Otorhinolaryngol Clin 2014;6(2):53-54.

Source of support: Nil

Conflict of interest: None

\section{INTRODUCTION}

Group A streptococcal (GAS) tonsillitis presents with abrupt onset of sore throat, tonsillar exudate, tender cervical adenopathy, and fever, followed by spontaneous resolution within 2 to 5 days. Streptococcal tonsillitis could result in various complications, such as suppurative (peritonsillar abscess, cervical lymphadenitis and mastoiditis) and nonsuppurative complications (acute rheumatic fever, glomerulonephritis, PANDAS syndrome). ${ }^{1}$

\footnotetext{
${ }^{1}$ Professor, ${ }^{2}$ Professor and Head, ${ }^{3}$ Postgraduate

${ }^{1-3}$ Department of ENT, JJM Medical College, Davangere Karnataka, India
}

Corresponding Author: UP Santosh, Professor, Department of ENT, JJM Medical College, Davangere, Karnataka, India, Phone: +91-9845155223, e-mail: drsantoshup@gmail.com
Although symptoms of GAS pharyngitis resolve without antibiotic therapy, persistence of the organisms in the upper respiratory tract elicits an immune response that can set the stage for subsequent risk of acute rheumatic fever (ARF) if the strain is rheumatogenic and the host is genetically predisposed. In some population, groups $G$ and $\mathrm{C}$ streptococci may also play a role in ARF pathogenesis. Evaluation of GAS tonsillitis therapies has been based upon GAS eradication from the upper respiratory tract; it is assumed that such eradication is an adequate surrogate marker for efficacy in primary prevention of rheumatic fever. ${ }^{2}$ Antibiotic therapy can be helpful for prevention of rheumatic fever if initiated up to 9 days following onset of symptoms.

Goals of antimicrobial therapy for eradication of GAS from the pharynx in the setting of acute streptococcal pharyngitis include the following:

- Reducing duration and severity of clinical signs and symptoms (suppurative complications).

- Reducing incidence of nonsuppurative complications (acute rheumatic fever).

- Reducing transmission to close contacts by reducing infectivity.

The present study aims at determining the frequency of antistreptolysin O (ASLO) positivity in cases of proven chronic tonsillitis.

\section{MATERIALS AND METHODS}

This is a time bound cross-sectional study done on 150 patients with clinically diagnosed chronic tonsillitis and willing for tonsillectomy at a tertiary health center over a period of 10 months from January 2013 to October 2013.

The patients ranged from 5 to 34 years (Table 1). A total of 70 patients were males and 80 females. On admission, a proforma was filled for each patient documenting age, sex, address, and clinical information, including chief complaints and duration of symptoms. Following this, a detailed otorhinolaryngological and general physical examination was done. Investigations including complete blood counts, bleeding time, clotting time, blood urea, serum creatinine HIV, HBsAg, ECG, chest X-ray, RF, ASLO titers (Table 2), and urine routine examination were done for all patients prior to surgery. Following the physicians and anesthetic fitness, 
Table 1: Distribution of cases according to the age

\begin{tabular}{ll}
\hline Age groups & No. of cases \\
\hline $5-14$ years & $70(46 \%)$ \\
$15-24$ years & $45(30 \%)$ \\
$25-34$ years & $35(24 \%)$ \\
\hline
\end{tabular}

Table 2: Distribution of cases with ASLO positivity

\begin{tabular}{ll}
\hline Age groups & No. of cases \\
\hline $5-14$ years & 2 \\
$15-24$ years & 2 \\
$25-34$ years & 1 \\
\hline
\end{tabular}

the patient was posted for tonsillectomy with or without adenoidectomy under general anesthesia and the tonsillar specimens were sent for histopathological examination.

Statistical analysis of results was carried out using statistical package for social science (SPSS) version 13. Level of significance is considered $<0.05$ for Chi-square test and $>0.4$ for kappa.

\section{RESULTS}

Majority of the patients were in the age group of 5 to 14 years $(46 \%)$. Overall, there was a female predominance.

Five out of the 150 patients had ASLO titer positivity $(>200 \mathrm{IU} / \mathrm{ml})$.

During a 6 month postoperative follow-up, three of the five cases showed negative ASLO titers.

\section{DISCUSSION}

ASLO testing is an essential tool in chronic tonsillitis as it can be used in the early detection of group A betahemolytic streptococcal tonsillitis as well as in the effective medical and surgical management of the same. ${ }^{3}$

Follow-up test of cure is appropriate for asymptomatic index patients and their asymptomatic household contacts in the following circumstances:

- Individuals with history of rheumatic fever

- Individuals who develop acute pharyngitis during an outbreak of acute rheumatic fever or acute poststreptococcal glomerulonephritis

- Spread of GAS among several family members.

In case of a recurrent streptococcal tonsillitis or serological positivity, they should receive a standard course of antimicrobial therapy (penicillin/1st generation cephalosporins). ${ }^{4}$ Repeated treatment should be administered with an agent with greater beta-lactamase stability than the previous agent. If penicillin was used for initial therapy, repeat treatment with amoxicillin-clavulanate or a 1st generation cephalosporin may be used; if initial treatment was with a 1st generation cephalosporin, a 2nd or 3rd generation cephalosporin may be used. ${ }^{5}$

The statistical conclusions made in our studies proved that serological testing in clinically proven cases of chronic tonsillitis could be detrimental in assessing the likelihood of GABS tonsillitis and their subsequent complications.

\section{CONCLUSION}

Early detection and treatment of streptococcal tonsillar involvement could be a preventive measure against the future predilection of other predisposed organs to more severe and debilitating streptococcal infections.

Further studies are required to ascertain the following:

1. ASLO titer estimation as a part of routine workup in chronic tonsillitis patients.

2. Would ASLO positivity become a relative indication for tonsillectomy?

3. Duration for post-tonsillectomy ASLO negativity.

4. Incidence of ASLO positive chronic tonsillitis and association with rheumatic fever, scarlet fever, poststreptococcal glomerulonephritis and PANDAS syndrome).

\section{REFERENCES}

1. Burmaa B, Zulgerel D, Bayarmaa D. Prevalence of streptococcus A tonsillitis and Rhuematic Fever among children in Ulaanbaatar city. WHO survey in Ulaanbaatar city; 2004.

2. Darrow DH, Siemens C. Indications for tonsillectomy and adenoidectomy. Laryngoscope 2002;112(8):6-10.

3. Viswanathan N, Nair SS, Thulseedharan S. Effect of tonsillectomy on ASLO titres. Indian J Otolaryngol Head Neck Surg 2000;52(4):329-331.

4. Brook I, Shah K. Bacteriology of adenoids and tonsil in children with recurrent adenotonsillitis. Ann Otol Rhinol Laryngol 2001;110(9):844-848.

5. Shulman ST, Tanz RR. Group A streptococcal pharyngitis and immune-mediated complications: from diagnosis to management. Expert Rev Anti infect Ther 2010;8(2);137-150. 